\title{
What can we do about tall girls?
}

An increasing number of parents now seek medical advice because they fear that their daughters will be too tall. Some of these girls have always been taller than their peers, but in others the tallness is of recent origin and is usually the result of a relatively early adolescent growth spurt which caused them to 'shoot up' above their classmates and possibly above an elder brother or sister. Sometimes tall parents of a very young child seek advice in the hope that something may be done to prevent their daughter from becoming as tall as they are.

The management of these cases involves three steps: (i) exclusion of pathological causes of tallness; (ii) prediction of the child's ultimate height; (iii) reassurance or, occasionally, treatment.

The pathological states associated with tallness are rare and are not usually difficult to recognize. Excessive growth hormone production may be accompanied by some features of acromegaly. In children and adolescents there are seldom any neurological symptoms or signs to suggest a pituitary adenoma but a tumour may be detected radiologically. In adult acromegalics the resting serum growth hormone is frequently raised and in some cases is not suppressed by raising the blood glucose level. In children, however, this test is difficult to interpret owing to lack of comparative data. A grossly abnormal result would presumably be good evidence of uncontrolled growth hormone production but the borderline between the normal and the abnormal is far from clear.

Hyperthyroidism, precocious puberty (with its several causes), and the feminizing testes syndrome should be considered in the differential diagnosis, as well as XXY and XYY karyotypes. Cerebral gigantism (Soto's syndrome) is diagnosed on clinical grounds. The hands and feet are big; the cranium and chin elongated; the lips thick and the eyes often wide apart with a slight antimongoloid slant. Though children with this condition may be very tall, their final stature is not always as great as one might expect from that during childhood. Patients with homocystinuria are clinically similar to those with Marfan's syndrome, though they are more likely to be of low intelligence, and the diagnosis must be made on biochemical grounds. The possibility of an abnormal karyotype (e.g. $\mathrm{XXY}$ or XYY) should also be considered.

In general, we may expect the daughter of tall parents to become a tall adult but the relation of the child's final height to that of either or both parents is not very close and there are many exceptions to the general rule. Also, most children who are tall after their third birthday are destined to become tall adults. Some of them, however, are tall in childhood only because they are early maturers. Their growth will stop at a relatively early age and before they become unusually tall. Neither the child's height alone nor that of her parents gives us enough information to make a prediction of her adult height on which we may base a decision about treatment. In addition to her height, we need information about the proportion of growth which has been completed. This is given, though not very precisely, by her bone age.

Tables for predicting a child's final height from her present height and bone age were published by Bayley (1946), and in their revised form (Bayley and Pinneau, 1952) for use with the bone age atlas of Greulich and Pyle (1959) are widely used for clinical purposes today. These tables have the apparent disadvantage that they do not allow the exact bone age to be taken into account but use only information as to whether it is average, advanced by 1 year, or delayed by 1 year. Also, the Atlas method of assessing bone age has certain theoretical disadvantages which are discussed by Tanner et al. (1975b) together with their alternative 'TW2' method of estimating bone age. Tanner et al. (1975a) have also published a new method of predicting adult height which takes into account not only the child's height and TW2 bone age, but also the occurrence of menarche and the average height of the two parents.

In general, errors of prediction using the method of Tanner et al., are slightly smaller than those arising from the Bayley-Pinneau method, and they are considerably smaller in postmenarcheal girls. Errors are further reduced when allowance is made for midparental height. Some paediatricians consider the Tanner method to be too laborious for 
clinical use, but a trained observer can estimate the bone age and calculate the predicted adult height within 10 minutes with the aid of a pocket calculator. It takes about the same time to use the Greulich/Pyle method accurately. Indeed, one of the drawbacks of this method is its apparent simplicity. It is very easy to use it badly and produce wildly inaccurate results. The step by step objective nature of the TW2 method precludes a slovenly approach.

In practice, neither method is ideal when applied to tall girls. Even the TW2 method carries an error of up to $\pm 6 \mathrm{~cm}$ in girls of normal stature aged 4 to 11 years. In $5 \%$ of cases the error may be greater. After this, the errors diminish with age and at age 13 are $\pm 4 \mathrm{~cm}$ for premenarcheal girls. The accuracy of both methods in predicting the final height of healthy girls whose statures are already above the 97th centile requires further investigation. However, the work of Roche and Wettenhall (1969) suggests that the Bayley and Pinneau method has about the same accuracy in tall girls as in shorter ones and this is probably true also of the TW2 method. Neither method is valid when applied to girls whose tallness is of pathological origin.

\section{Treatment}

The management of those pathological states which may be associated with tallness will not be considered here. When active treatment is possible it must be aimed at the underlying disorder.

Let us assume that we are faced with a perfectly healthy 10-year-old girl whose predicted adult height is 5 feet 11 inches $(180 \mathrm{~cm})$. If we allow for errors of prediction, she might reach 6 feet $(183 \mathrm{~cm})$ or even 6 feet 1 inch $(185 \mathrm{~cm})$. On the other hand, she might only reach 5 feet 9 inches $(175 \mathrm{~cm})$. If we repeat the prediction two or three times at 6-monthly intervals we may be able to make a subjective judgement as to whether the initial error was on the high or the low side, but we shall still be uncertain about the outcome.

If we were able to offer a simple and absolutely safe treatment which could be guaranteed to reduce the final height by 2 or 3 inches, the parents would probably wish us to use it. Unfortunately, we are not in this happy position. The only active treatments we can offer are oestrogens and surgery.

Oestrogens may accelerate the maturation of the skeleton and thereby reduce the time for which growth can continue. In some cases they may also reduce the speed of growth. The exact mechanism of oestrogen action is not clear. They do not reduce the rate of growth hormone production but ap- parently enhance it (Plotnick et al., 1974), and high blood levels of growth hormone have been found in tall girls under treatment though the somatomedin levels were low. Weidemann and Schwartz (1972) have also shown suppression of somatomedin by oestrogen. This action would explain reduction in growth velocity by oestrogens but not the concurrent acceleration of skeletal maturation, which implies an androgenic effect. Zachmann et al. (1975) have suggested that the large doses of oestrogen used for the treatment of tall girls may act on the skeleton indirectly by increasing the output of androgens from the adrenals. They have reported a mean reduction in adult height (as compared with TW2 prediction) of $4.6 \mathrm{~cm}$ in 40 girls, though no difference between final and predicted height was found in 3 of these patients. They gave ethinyl estradiol $0.3 \mathrm{mg}$ daily to 29 of these cases, with norethisterone $10 \mathrm{mg}$ daily for 5 to 7 days every fourth week. The remaining 11 cases were treated with stilboestrol $3 \mathrm{mg}$ daily. The mean duration of treatment was $1 \cdot 7$ years.

Several other authors using various oestrogen preparations have reported reduction in final height similar to or less than this, when compared with the predicted height before treatment. Thus, we can offer treatment to our patient which is easy to take and which may lead to her final height being up to $2 \frac{1}{2}$ inches $(6 \mathrm{~cm})$ less than our prediction but may have no apparent effect at all. We cannot say that it is absolutely safe.

The association between oral contraceptives and thromboembolism is well known, though it has not been observed in girls treated with oestrogen to reduce their stature. Hypertension and liver damage are other possible side effects, and we do not know whether or not some impairment of fertility may result. Some girls have become pregnant after treatment but the majority of treated subjects are not yet married. Theoretically the risks are considerable but it appears that they seldom materialize.

Clearly the balance between 'pros' and 'cons' of oestrogen therapy is a fine one, and treatment should not be undertaken unless the parents fully understand all the implications and are convinced that the risks are outweighed by the threat to their child's future happiness if nothing is done. The author is seldom prepared to offer treatment even on these terms unless serial predictions indicate that the patient's final stature may exceed 6 feet $(183 \mathrm{~cm})$. However, one cannot make absolute rules.

Ethinyl oestradiol as used by Zachmann et al. is probably the most satisfactory regimen, though a 
lower dosage may be equally effective. Conjugated oestrogens contain substances of unknown effect, while stilboestrol sometimes causes unsightly enlargement and pigementation of the areola. Oestradiol valerate is relatively free from side effects but it must be given by injection.

It is reasonable to expect the effect of the treatment to diminish when it is begun at later ages. Hence, we could recommend that it should begin at the earliest age when growth of the breasts would be acceptable. However, the results of Zachmann et al. suggest that the age at which treatment begins may not greatly affect the results and that treatment even after menarche may be effective. These authors suggest that there may be psychological advantages in delaying treatment until the natural development of the breasts has begun. However, it should be remembered that we never know exactly how effective the treatment has been in an individual case and can only refer to our prediction with its inherent error.

The alternative treatment, by surgical shortening of the limbs, is clearly a greater immediate stress for the patient than taking a daily oestrogen pill, but has the great advantage that it can be deferred until the patient's adult height is known and she has discovered whether or not it is a sufficient handicap to justify the procedure. If her legs are relatively long in relation to the trunk (as they frequently are in tall girls) the surgery need not result in any apparent disproportion. Orthopaedic surgeons differ in their attitude to this form of treatment and many would be unwilling to undertake it except in the most extreme circumstances.

Oestrogen therapy is justified in a small number of very tall girls who are so distressed by their growth prospects that a possibility of losing even an inch or two from their final height is a great comfort to them. It should only be undertaken after the fullest possible consultation with the parents and the child herself, and under the guidance of someone experienced in the assessment of growth. Perhaps in the future somatostatin may have a role in the treatment of these patients.

\section{REFERENCES}

Bayley, N. (1946). Tables for predicting adult height from skeletal age and present height. Fournal of Pediatrics, 28, 49.

Bayley, N., and Pinneau, S. R. (1952). Tables for predicting adult height from skeletal age. Fournal of Pediatrics, 40, 423.

Greulich, W. W., and Pyle, S. I. (1959). Radiographic Atlas of Skeletal Development of the Hand and Wrist. 2nd ed. Stanford University Press, California.

Plotnick, I. P., Thompson, R. G., Beitins, I., and Blizzard, R. M. (1974). Integrated concentrations of growth hormone correlated with stage of puberty and estrogen levels in girls. fournal of Clinical Endocrinology and Metabolism, 38, 436.

Roche, A. F., and Wettenhall, H. N. B. (1969). The prediction of adult stature in tall girls. Australian Paediatric fournal, 5, 13.

Tanner, J. M., Whitehouse, R. H., Marshall, W. A., and Carter, B. S. (1975a). Prediction of adult height from height, bone age, and occurrence of menarche at ages 4 to 16 with allowance for midparent height. Archives of Disease in Childhood, 50, 14.

Tanner, J. M., Whitehouse, R. H., Marshall, W. A., Healy, M. J. R., and Goldstein, H. (1975b). Assessment of Skeletal Maturity and Prediction of Adult Height: T.W.2 Method. Academic Press, New York. (In press.)

Weidemann, E., and Schwartz, E. (1972). Suppression of growth hormone-dependent human serum sulfation factor by estrogen. fournal of Clinical Endocrinology and Metabolism, 34, 51.

Zachmann, M. Ferrandez, A., Mürset, G., and Prader, A. (1975). Estrogen treatment of excessively tall girls. Helvetica Paediatrica Acta, 30, 11.

W. A. MARshaLI

Department of Growth and Development, Institute of Child Health, 30 Guilford Street, London WC1N 1EH. 